of some electromagnetic analogy. How to turn it into a theory is another matter. Besides, another mechanism still has to be found to explain Leipuner's data at low transverse momentum, where vector mesons can only account for $10 \%$ of the observed muon yield. The decay of the $\eta$ meson (a kind of heavy neutral pion) into two muons and a photon might account for another $15 \%$ in this case, but would upset the observed balance between electrons and muons if used to explain the large transverse momentum data.

Unfortunately, lack of data on the production of unstable particles prevents us dismissing out of hand the unlikely possibility that known heavier relatives of the $\rho^{0}, \omega^{0}$ and $\varphi^{0}$ give rise to the bulk of the muons and electrons. They are included in the generic title "heavy virtual photons' given to the unknown parents.

An assortment of ordinary mechanisms would not satisfy the discoverers of direct muons, whose searches had a more glamorous stimulus. Theorists have found it necessary to postulate the existence of many new particles in order to account for various features of the weak interaction, more than just the field particles needed to carry the weak force. Those ideas have gained more support with the recent discovery of neutral weak currents.

According to one class of theories, there should be a whole set of new strongly interacting particles with a new quantum number 'charm'. There could even be a fourth degree of freedom, 'colour', to add to isotopic spin, strangeness and charm and give rise to its own family of particles. Strong decays of the new particles would be inhibited by selection rules, giving plenty of opportunity for 'direct' muons and electrons to be produced by weak or electromagnetic decays.

The $J$ and $\psi$ particles may fit such a scheme, but if their production is as infrequent as is rumoured they cannot

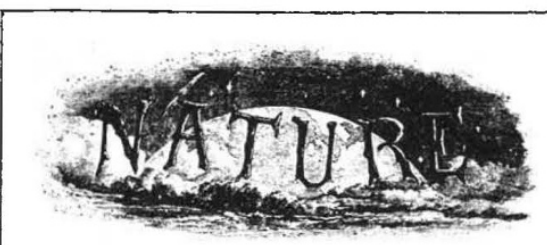

\section{A hundred years ago}

A METEOR was not only seen but actually caught at Orleans on the 9th inst. A small mass of pyritous substance was discovered in one of the streets, at the very place which had been struck by an immense flame a few seconds before. The pieces were divided among bystanders anxious to secure the possession of the smallest fragment of such a celestial object; but it is hoped some of the possessors will be intelligent enough to get a specimen sent to the Academy of Sciences.

from Nature, 11, 396; March 18, 1875 be the source of so many direct muons. Are there others, with masses low enough to be produced in $28 \mathrm{GeV}$ proton-uranium collisions?

We can expect many results bearing on these questions in the next few months. A new impetus has been given to attempts to measure the production characteristics of the well known unstable particles. The complete results of Ting's J-particle experiment should answer some of the mundane questions as well as the more exciting ones. Every high energy laboratory is mounting programmes to search for charm and colour. Also, many groups are looking again at their 'zoos' of supposed artefacts, conscious that neutral currents, J-particles and the direct muons all gave signs of their presence in earlier experiments, though too faint to create universal enthusiasm for following them up.

\section{Polyacrylamide gels}

\section{from Paul Calvert}

Cross-Linked polyacrylamide gels are extensively used in the electrophoretic separation of proteins. It is thought that the gel acts as a molecular sieve with a distribution of pore sizes in the $20 \AA$ range so that small molecules pass easily through and large molecules are retarded. These gels are prepared by the free radical polymerisation of an aqueous solution of a few per cent acrylamide containing a small amount of cross-linking agent. Under these conditions one might reasonably expect to get a homogeneous network. Pore sizes calculated on this assumption (see for instance Robard and Chrombach, Proc. natn. Acad. Sci. U.S.A., 65, 970; 1970; Hersey and Rees, Nature phys. Sci., 230, $92 ; 1971)$ are about right for protein dimensions and thus we have a fine example of a simple theory giving good results.

Unfortunately for lovers of simple theories, recent scanning electron microscopy of freeze dried gels suggests that the structure is not homogeneous at all. Blank and Reimschuessel ( $J$. Materials Sci., 9, 1815; 1974) studied similar polyacrylamide gels from the point of view of their suitability as support media for crystal growth. Their results show a structure consisting of $2-10 \mu \mathrm{m}$ walls, surrounding pores of similar dimensions, in a 2.5 weight per cent acrylamide gel. As the concentration is increased the walls become thicker. This size is suitable for the growth of crystals but huge compared to any protein molecule so that if there is a sieving action it must be within the walls, and will depend on the pores not being interconnected.

First we must consider whether the pores are an artefact of the preparation technique. The gels were prepared by ${ }^{60} \mathrm{Co}$ irradiation rather than by a catalyst-accelerator system but this should not be a crucial difference. The usual concentration of cross-linking agent was used. The freeze drying may have distorted the structure but the kind of large-scale chain breakage needed to create the pores seems unlikely. Further, a cross-linked polyvinyl alcohol gel showed only very small pores after freeze drying.

There have been many reports of anomalous behaviour of organic gels. In gelatin gels Johnson and Thornton (J. Photog. Sci., 16, 117; 1968) showed that $1 \mu \mathrm{m}$ particles have considerable mobility. Pines and Prins (Macromolecules, 6, 888; 1973) have demonstrated phase separation in agarose and some polyvinyl alcohol gels but not in gelatin.

Thus there is some evidence for a macroscopic pore structure in several gel systems which must arise by a liquid-liquid phase separation process analogous to that observed in many polymer solutions below the theta temperature (see Flory's Principles of Polymer Chemistry) but with the additional constraint of the cross-linked structure to be accounted for. In terms of the electrophoretic separation the presence of the macroscopic pores cannot do much good and it seem that further study of the relationship between structure and separation could lead to improved gel systems.

\section{Changes in species diversity}

\section{from Peter D. Moore}

IF there is one area of ecology in which theoretical speculation has outpaced acquisition of field data it is in the study of succession. The time scale involved in most terrestrial successions is of the order of centuries, which leaves the hopeful student with limited options: he must either be contented with the study of a series of extant phases which are assumed to be related in a successional series, or he must turn to systems which attain a more rapid equilibrium (such as plankton) for the formulation or confirmation of generalised models. Neither course is satisfactory, but life is short.

The former alternative has recently been chosen by Nicholson and Monk (Ecology, 55, 1075; 1974) as a means of investigating the vexed problem of diversity changes during succession. By obtaining data on the vascular plant composition of 51 seral vegetation types developed on abandoned agricultural land in the Georgia Piedmont, they have produced one of the most extensive sets of information currently avail- 